\title{
Reduction and technical simplification of testing protocol for walking based on repeatability analyses: An Interreg IVa pilot study
}

\author{
Nejc Sarabon (1,2), Stefan Loefler (1), Hannah Fruhmann (1), Samantha Burggraf (1),
} Helmut Kern (1)

(1) Ludwig Boltzmann Institute of Electrical Stimulation and Physical Rehabilitation, Vienna, Austria; (2) University of Primorska, Science and Research Centre, Koper, Slovenia.

\begin{abstract}
The aim of this study was to define the most appropriate gait measurement protocols to be used in our future studies in the Mobility in Ageing project. A group of young healthy volunteers took part in the study. Each subject carried out a 10-metre walking test at five different speeds (preferred, very slow, very fast, slow, and fast). Each walking speed was repeated three times, making a total of 15 trials which were carried out in a random order. Each trial was simultaneously analysed by three observers using three different technical approaches: a stop watch, photo cells and electronic kinematic dress. In analysing the repeatability of the trials, the results showed that of the five self-selected walking speeds, three of them (preferred, very fast, and very slow) had a significantly higher repeatability of the average walking velocity, step length and cadence than the other two speeds. Additionally, the data showed that one of the three technical methods for gait assessment has better metric characteristics than the other two. In conclusion, based on repeatability, technical and organizational simplification, this study helped us to successfully define a simple and reliable walking test to be used in the main study of the project.
\end{abstract}

Key Words: walking, gait, repeatability, measurement, evaluation

European Journal Translational Myology - Basic Applied Myology 2010; 1 (4): 181-186

Human upright posture, bipedal stance, and gait are unique among mammals [6]. They represent an important evolutionary milestone $[15,26]$ that resulted in (i) significantly increased balance requirements and accompanied development of the brain and vestibular system [6], (ii) characteristic inter-segmental and intermuscular coordination during movement [4], and (iii) freeing upper extremities and their reciprocal cyclic movement pattern during walking gait [28]. From the biomechanical point of view, human gait is a marvel, involving repeatedly losing and then re-gaining one`s balance [10]. Studies on mechanics of locomotion have covered a wide spectrum from largely speculative to strictly quantitative. Already from the pioneering studies of human locomotion onwards, there was a distinction between these two methodological approaches of their validation [12].

The purpose for which one would like to evaluate gait and the availability of suitable measurement equipment define the limits of complexity of the measurement process. Several research disciplines have contributed to today's thorough understanding of human gait from the biomechanical, functional-anatomical, and motor control point of view [14]. Technological advancements now enable researchers to make measurements more precisely and to make more complex analyses. In clinical research practice we also need to optimize the measurement protocol so that we can gather valid, objective, and repeatable information. From a cost-benefit point of view, we should seek to use the least time, both of human resources and of technical equipment. We should also avoid impairment of the results in the study protocol due to fatigue on the part of the subjects undergoing the tests.

Three basic parameters which characterize walking are speed, cadence, and step length [13,25]. The interplay among these has been analysed using kinematics, accelerometers and other measurement methods [2,5,9, $17,21,24,27]$.

The purpose of this study was to define the gait measurement tests for the main cross-sectional study and the intervention studies which will follow in the later stages of our project (Mobility in Aging, InterregIva Austria-Slovakia, SK-AT_080612_N00033). In 
Gait and balance in young and elderly

European Journal Translational Myology - Basic Applied Myology 2010; 1 (4): 181-186

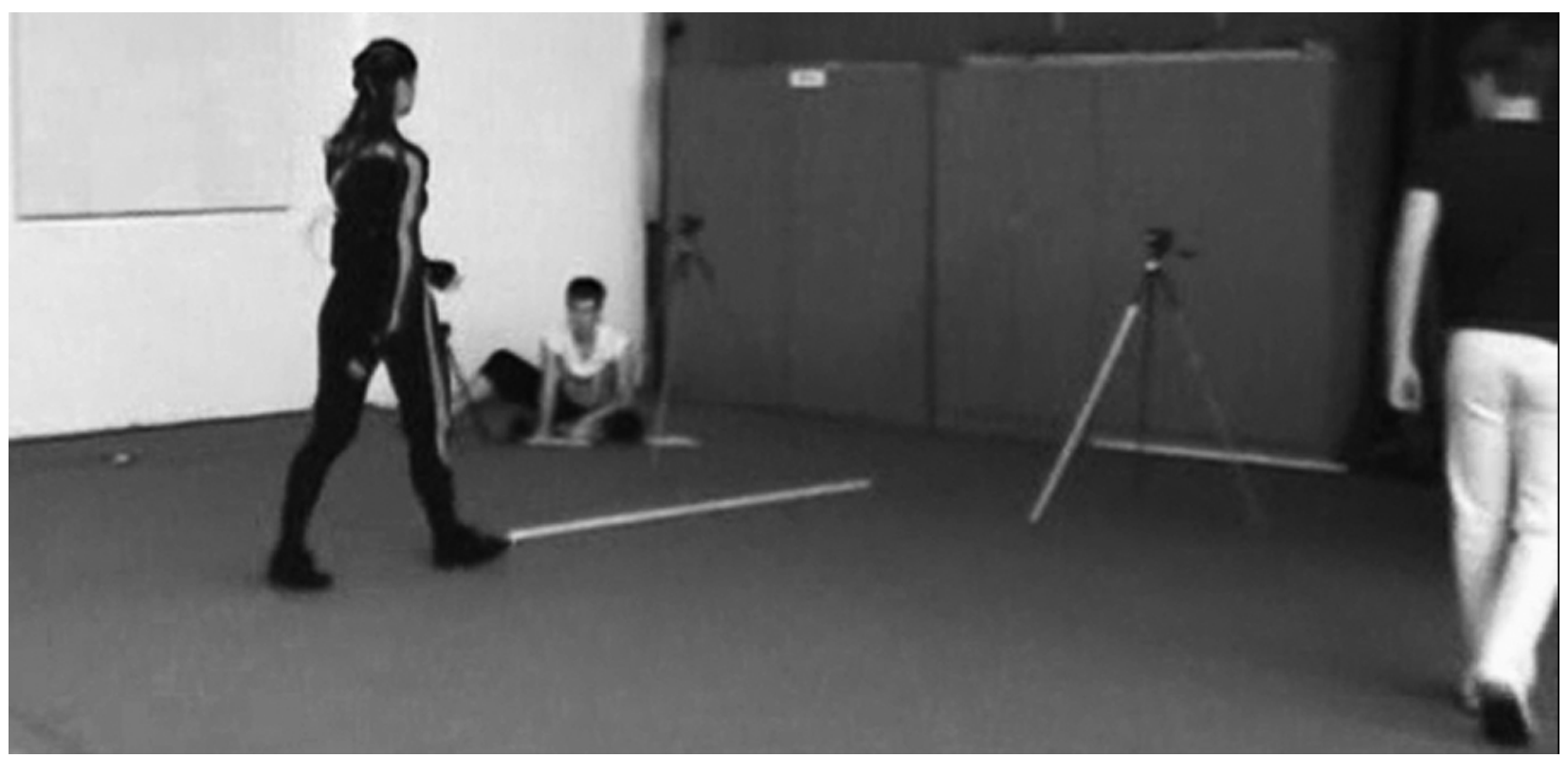

Fig 1. Measurement set-up: (i) a subject in the MOV dress that wirelessly transfers the data to the computer which was operated by the first measurer, (ii) the sitting measurer was noting down the time acquired by the photocells and made additional precise measurements of the last remaining step using the measurement tape, (iii) the third measurer was only estimating the length of the last step and measuring the time with the manual stop-watch.

following this aim, we carried out tests and analyses with which we compared the repeatability of the three main parameters of gait when using different speeds of walking and/or different technical assessment methods. Based on the criteria of reliability, accuracy and technical simplicity we made a proposal for the main study that will be carried out on a larger number of young and also of elderly subjects.

\section{Materials and Methods}

\section{Subjects}

At the outset, the protocol of the study, approved by the national committee for medical ethics, was explained to the potential participants. Sixteen young healthy subjects ( 9 male, 7 female; age $25.9 \pm 4.8$ years; body height $176.9 \pm 8.4 \mathrm{~cm}$; body weight $71.4 \pm$ $12.4 \mathrm{~kg}$ ) volunteered for the study and signed a full informed consent document prior to their enrolment.

\section{Measurements}

The subjects were asked to walk a 10-metre distance with five different speeds: (i) preferred speed, i.e. the self-chosen normal speed of walking, (ii) very fast speed, i.e. the fastest walking speed, but not running, (iii) a very slow speed, i.e. the slowest possible walking, without stopping the movement at any time, (iv) fast speed, i.e. walking at the mid-speed between the preferred and the very fast, and (v) slow speed, i.e. walking at the mid-speed between the preferred and very slow. Each individual of this group performed three repetitions of walking at each speed. This makes altogether 15 walking repetitions, which were carried out in a random order. Rest intervals of $60 \mathrm{~s}$ were used between the consecutive trials in order to avoid the development of fatigue. Parameters of gait in every 10meter walking trial were calculated using three different methods: (i) a special measurement dress with embedded electronic sensors aimed to precisely acquire data on the dynamics of body motion (XsenseMoven, Enschede, Netherlands) - MOV, (ii) a combination of photo cells at the start and the finish of the 10-metre walking track, counting the steps during visual inspection, and measuring the length of the remaining part of the last step made at the end of the walking track - PCM, and (iii) a combination of a standard stop-watch instead of photo cells, counting the number of steps and only subjective estimation of the remained part of the last step using $10 \mathrm{~cm}$ precision - SWE. Each of the measurement methods was carried out by a dedicated examiner, thus, three examiners were measuring the same trial at the same time (Figure 1). For each walking trial the following parameters were calculated: average walking speed, average step length and average step cadence.

\section{Analysis}

For statistical analyses SPSS 17 software (SPSS Inc., Chicago, USA) was used. Descriptive statistics were calculated for all sets of the data, and represented as mean \pm standard deviation. Then, a test-retest repeatability analysis was performed for all the 


\section{Gait and balance in young and elderly}

European Journal Translational Myology - Basic Applied Myology 2010; 1 (4): 181-186

parameters. Intra-class correlation coefficients were calculated for a single trial (ICCs) and the average of three trials (ICCa); 95\% confidence intervals (CI) were calculated. One-way ANOVA was used to test for bias

Table 1: Comparison of repeatability in the context of walking speed (5 speeds) and measurement method (3 methods). The top horizontal cluster shows means and standard deviations $(A V G \pm S D)$ followed by the repeatability parameters (ICCs, ICCa, CI, SEM, and ANOVA $(F, p)$ ) for average walking speed (A), average step length (B) and average step cadence $(C)$. The underlined values in the last cluster highlights the statistically significant ANOVA values (i.e. inter-trial significant differences) $(p<0.05)$.

\begin{tabular}{|c|c|ccc|}
\hline \multirow{2}{*}{ TABLE 1A } & \multicolumn{3}{c|}{ WALKING SPEED } \\
\cline { 2 - 5 } & very slow & $0.56 \pm 0.16 \mathrm{~m} / \mathrm{s}$ & $0.56 \pm 0.16 \mathrm{~m} / \mathrm{s}$ & $0.58 \pm 0.16 \mathrm{~m} / \mathrm{s}$ \\
& slow & $1.01 \pm 0.10 \mathrm{~m} / \mathrm{s}$ & $1.01 \pm 0.10 \mathrm{~m} / \mathrm{s}$ & $1.02 \pm 0.10 \mathrm{~m} / \mathrm{s}$ \\
\multirow{3}{*}{ AVG \pm SD } & prefered & $1.40 \pm 0.13 \mathrm{~m} / \mathrm{s}$ & $1.38 \pm 0.12 \mathrm{~m} / \mathrm{s}$ & $1.41 \pm 0.12 \mathrm{~m} / \mathrm{s}$ \\
& fast & $1.84 \pm 0.16 \mathrm{~m} / \mathrm{s}$ & $1.78 \pm 0.14 \mathrm{~m} / \mathrm{s}$ & $1.82 \pm 0.15 \mathrm{~m} / \mathrm{s}$ \\
& very fast & $2.29 \pm 0.22 \mathrm{~m} / \mathrm{s}$ & $2.22 \pm 0.19 \mathrm{~m} / \mathrm{s}$ & $2.27 \pm 0.20 \mathrm{~m} / \mathrm{s}$ \\
\hline \multirow{3}{*}{ ICCs/CI } & very slow & $0.72 / 0.46-0.89$ & $0.80 / 0.59-0.92$ & $0.77 / 0.55-0.91$ \\
& slow & $0.40 / 0.09-0.71$ & $0.41 / 0.11-0.72$ & $0.40 / 0.10-0.71$ \\
& prefered & $0.74 / 0.50-0.90$ & $0.81 / 0.60-0.93$ & $0.78 / 0.56-0.92$ \\
& fast & $0.79 / 0.57-0.92$ & $0.78 / 0.55-0.92$ & $0.80 / 0.59-0.92$ \\
& very fast & $0.86 / 0.70-0.95$ & $0.87 / 0.72-0.95$ & $0.87 / 0.71-0.95$ \\
\hline \multirow{3}{*}{ ICCa/CI } & very slow & $0.88 / 0.72-0.96$ & $0.92 / 0.81-0.97$ & $0.91 / 0.78-0.97$ \\
& slow & $0.67 / 0.24-0.88$ & $0.68 / 0.27-0.89$ & $0.67 / 0.24-0.88$ \\
& prefered & $0.90 / 0.75-0.96$ & $0.93 / 0.82-0.97$ & $0.91 / 0.79-0.97$ \\
& fast & $0.92 / 0.80-0.97$ & $0.92 / 0.79-0.97$ & $0.92 / 0.81-0.97$ \\
& very fast & $0.95 / 0.87-0.98$ & $0.95 / 0.88-0.98$ & $0.95 / 0.88-0.98$ \\
\hline \multirow{3}{*}{ SEM/F/p } & very slow & $0.09 / 0.44 / 0.65$ & $0.10 / 1.25 / 0.30$ & $0.09 / 0.78 / 0.47$ \\
& slow & $0.21 / 4.99 / \underline{0.01}$ & $0.19 / 4.55 / \underline{0.02}$ & $0.20 / 4.73 / \underline{0.02}$ \\
& prefered & $0.07 / 0.89 / 0.42$ & $0.03 / 0.18 / 0.83$ & $0.05 / 0.63 / 0.54$ \\
& fast & $0.11 / 2.20 / 0.13$ & $0.13 / 3.85 / \underline{0.03}$ & $0.12 / 3.21 / \underline{0.06}$ \\
& very fast & $0.03 / 0.28 / 0.76$ & $0.03 / 0.16 / 0.85$ & $0.03 / 0.24 / 0.79$ \\
\hline
\end{tabular}

\begin{tabular}{|c|c|ccc|}
\hline \multicolumn{2}{|c}{ TABLE 1B } & \multicolumn{3}{l|}{ STEP LENGTH } \\
\cline { 2 - 5 } & very slow & $0.55 \pm 0.06 \mathrm{~m}$ & $0.55 \pm 0.06 \mathrm{~m}$ & $0.55 \pm 0.06 \mathrm{~m}$ \\
& slow & $0.66 \pm 0.04 \mathrm{~m}$ & $0.66 \pm 0.04 \mathrm{~m}$ & $0.65 \pm 0.04 \mathrm{~m}$ \\
\multirow{3}{*}{ AVG \pm SD } & prefered & $0.76 \pm 0.05 \mathrm{~m}$ & $0.77 \pm 0.04 \mathrm{~m}$ & $0.76 \pm 0.05 \mathrm{~m}$ \\
& fast & $0.88 \pm 0.07 \mathrm{~m}$ & $0.88 \pm 0.06 \mathrm{~m}$ & $0.88 \pm 0.07 \mathrm{~m}$ \\
& very fast & $0.97 \pm 0.08 \mathrm{~m}$ & $0.97 \pm 0.08 \mathrm{~m}$ & $0.96 \pm 0.08 \mathrm{~m}$ \\
\hline \multirow{3}{*}{ ICCs/CI } & very slow & $0.90 / 0.77-0.96$ & $0.89 / 0.75-0.96$ & $0.89 / 0.76-0.96$ \\
& slow & $0.50 / 0.18-0.78$ & $0.47 / 0.15-0.75$ & $0.52 / 0.20-0.79$ \\
& prefered & $0.74 / 0.50-0.90$ & $0.80 / 0.58-0.92$ & $0.81 / 0.61-0.93$ \\
& fast & $0.86 / 0.71-0.95$ & $0.85 / 0.68-0.94$ & $0.86 / 0.70-0.95$ \\
& very fast & $0.87 / 0.71-0.95$ & $0.85 / 0.69-0.94$ & $0.87 / 0.72-0.95$ \\
\hline \multirow{3}{*}{ ICCa/CI } & very slow & $0.96 / 0.91-0.99$ & $0.96 / 0.90-0.99$ & $0.96 / 0.90-0.99$ \\
& slow & $0.75 / 0.41-0.91$ & $0.72 / 0.35-0.90$ & $0.76 / 0.43-0.92$ \\
& prefered & $0.90 / 0.75-0.96$ & $0.92 / 0.81-0.97$ & $0.93 / 0.82-0.97$ \\
& fast & $0.95 / 0.88-0.98$ & $0.94 / 0.86-0.98$ & $0.95 / 0.87-0.98$ \\
& very fast & $0.95 / 0.88-0.98$ & $0.95 / 0.87-0.98$ & $0.95 / 0.89-0.98$ \\
\hline \multirow{3}{*}{ SEM/F/p } & very slow & $0.03 / 0.76 / 0.48$ & $0.03 / 1.50 / 0.24$ & $0.03 / 1.50 / 0.24$ \\
& slow & $0.06 / 4.89 / 0.02$ & $0.07 / 4.95 / 0.01$ & $0.07 / 5.20 / 0.01$ \\
& prefered & $0.03 / 1.42 / 0.26$ & $0.00 / 0.37 / 0.69$ & $0.00 / 0.77 / 0.47$ \\
& fast & $0.03 / 1.83 / 0.18$ & $0.03 / 1.36 / 0.28$ & $0.03 / 1.77 / 0.19$ \\
& very fast & $0.00 / 0.48 / 0.63$ & $0.00 / 0.45 / 0.64$ & $0.00 / 0.36 / 0.70$ \\
\hline
\end{tabular}


Gait and balance in young and elderly

European Journal Translational Myology - Basic Applied Myology 2010; 1 (4): 181-186

effect between the three trials of the same task. Standard error of measurement (SEM) was calculated. The level of statistical significance was set at 0.05.

\section{Results and Discussion}

All the results are presented in Table 1. The five different subjectively defined speeds of walking turned out to be clearly distinguishable. This can be seen already from the mean values of all three observed parameters and was further confirmed by the statistically significant differences (ANOVA $\mathrm{p}<0.05$ ). A linear progression of walking speed was mirrored in a parallel increase in step length and step cadence. All these observations were independent of the measurement technique used.

Repeatability analyses showed comparable values of ICCs and ICCa among the three measurement methods. However, slightly higher ICC values were found for PCM. The repeatability of a single trial was in the low-to-moderate range. ICCa reached values that indicate a very good repeatability (ICCa $0.90-1.00$ ) for all but the slow speed of walking. In the latter case only step cadence reached high ICCa values (0.91), while those of the walking speed and step length remained low (0.68 and 0.72, respectively).

No inter-trial bias effect was observed for the preferred, very fast, and very slow speeds of walking (ANOVA p > 0.05). But, in the case of slow and fast speed, in several cases such a systematic inter-trial difference was present. For the same speeds (slow and fast) also the SEM values were obviously higher than in the case of the other three walking speeds.
A key feature of our independence as human beings is the ability to move from one place to another, i.e. mobility. For this aim humans predominantly use walking as one of the main gaits of locomotion. It is an extraordinarily complex behaviour that comprises three indispensable components: progression, postural control, and adaptability [22]. Walking speed is a function of step length and step frequency or cadence. Humans have a preferred speed of locomotion, which is guided by a combination of metabolic and mechanical factors [23]. Vast number of studies has been dedicated to the analyses of different aspects of human locomotion (for review see $[7,10,16,20]$ ), but it remains still one of the basic functional movements analysed in clinical studies. In spite of all the technological advancements, time-, cost-, and human resources optimization remain a great challenge for many clinical studies. Additionally, we also need to think of the unwanted effects of fatigue, lack of attention and motivation, etc., in this context, especially when patients and/or elderly subjects are involved.

The results of this study showed that of the five selfselected walking speeds, three of them (preferred, very fast, and very slow) had significantly higher repeatability of average walking speed, step length and cadence than the remaining two (fast and slow). Additionally, the data showed that PCM, one of the three technical methods for gait assessment (photo cells, counting of the steps, and measurement of the remaining part of the last step) gave better results than either of the other two methods. The final aim of our

\begin{tabular}{|c|c|ccc|}
\hline \multirow{2}{*}{ TABLE 1C } & \multicolumn{3}{c|}{ STEP CADENCE } \\
\cline { 3 - 5 } & very slow & $1.01 \pm 0.26 \mathrm{st} / \mathrm{s}$ & $1.01 \pm 0.26 \mathrm{st} / \mathrm{s}$ & $1.05 \pm 0.27 \mathrm{st} / \mathrm{s}$ \\
& slow & $1.53 \pm 0.14 \mathrm{st} / \mathrm{s}$ & $1.52 \pm 0.13 \mathrm{st} / \mathrm{s}$ & $1.57 \pm 0.14 \mathrm{st} / \mathrm{s}$ \\
\multirow{3}{*}{ AVG \pm SD } & prefered & $1.84 \pm 0.16 \mathrm{st} / \mathrm{s}$ & $1.80 \pm 0.12 \mathrm{st} / \mathrm{s}$ & $1.86 \pm 0.15 \mathrm{st} / \mathrm{s}$ \\
& fast & $2.08 \pm 0.12 \mathrm{st} / \mathrm{s}$ & $2.01 \pm 0.11 \mathrm{st} / \mathrm{s}$ & $2.08 \pm 0.13 \mathrm{st} / \mathrm{s}$ \\
& very fast & $2.36 \pm 0.16 \mathrm{st} / \mathrm{s}$ & $2.30 \pm 0.18 \mathrm{st} / \mathrm{s}$ & $2.37 \pm 0.17 \mathrm{st} / \mathrm{s}$ \\
\hline \multirow{3}{*}{ ICCs/CI } & very slow & $0.58 / 0.27-0.82$ & $0.77 / 0.54-0.91$ & $0.75 / 0.51-0.90$ \\
& slow & $0.65 / 0.36-0.85$ & $0.73 / 0.48-0.90$ & $0.72 / 0.47-0.89$ \\
& prefered & $0.76 / 0.53-0.91$ & $0.87 / 0.72-0.95$ & $0.87 / 0.71-0.95$ \\
& fast & $0.71 / 0.46-0.89$ & $0.74 / 0.50-0.90$ & $0.79 / 0.57-0.92$ \\
& very fast & $0.89 / 0.76-0.96$ & $0.91 / 0.79-0.97$ & $0.91 / 0.80-0.97$ \\
\hline \multirow{3}{*}{ ICCa/CI } & very slow & $0.81 / 0.52-0.93$ & $0.91 / 0.78-0.97$ & $0.90 / 0.76-0.97$ \\
& slow & $0.85 / 0.63-0.95$ & $0.89 / 0.74-0.96$ & $0.89 / 0.72-0.96$ \\
& prefered & $0.91 / 0.77-0.97$ & $0.95 / 0.89-0.98$ & $0.95 / 0.88-0.98$ \\
& fast & $0.88 / 0.72-0.96$ & $0.90 / 0.75-0.96$ & $0.92 / 0.80-0.97$ \\
& very fast & $0.96 / 0.91-0.99$ & $0.97 / 0.92-0.99$ & $0.97 / 0.92-0.99$ \\
\hline \multirow{3}{*}{ SEM/F/p } & very slow & $0.05 / 0.21 / 0.81$ & $0.05 / 0.34 / 0.72$ & $0.05 / 0.28 / 0.76$ \\
& slow & $0.15 / 3.15 / 0.06$ & $0.12 / 2.81 / 0.08$ & $0.14 / 3.20 / 0.06$ \\
& prefered & $0.12 / 2.04 / 0.15$ & $0.03 / 0.61 / 0.55$ & $0.07 / 1.64 / 0.21$ \\
& fast & $0.08 / 1.51 / 0.24$ & $0.09 / 2.48 / 0.10$ & $0.09 / 2.42 / 0.11$ \\
& very fast & $0.03 / 0.09 / 0.92$ & $0.00 / 0.04 / 0.96$ & $0.03 / 0.07 / 0.94$ \\
\hline
\end{tabular}




\section{Gait and balance in young and elderly}

European Journal Translational Myology - Basic Applied Myology 2010; 1 (4): 181-186

project is obtain reliable results from a wide-ranging study not only of young adults but also of healthy elderly and also of frail elderly subjects. Based on the observed reliability parameters, organizational simplification, money-for-value, we found that the PCM method is the best choice.

The primary aim of this pilot study was to establish the final measurement protocol for a quick, but informative analysis of walking which will serve in the following studies of the project, to establish normative values for different groups of subjects (defined by age and level of physical fitness). Besides the already mentioned optimization criteria, we have primarily analysed the repeatability measures of the tests/parameters. Precision and repeatability are namely of crucial importance for each measurement to be practically useful. However, we should not forget that human biological systems which control walking movement patterns include variability as an intrinsic characteristic [18]. From this point of view, we should distinguish between noise variability and functional variability. In this study we were primarily interested in minimizing the noise, thereby looking at its random as well as the systematic components [19]. But, in our further study, we will analyse also the inter- and intraindividual differences in variability of the movement pattern which are both functionally very relevant in the context of dynamic systems theory [11].

Several recent studies $[1,3,8]$ have shown that the intrinsic variability of the gait parameters (stride time, stride length, joint kinematics, etc.) is dependent on gait velocity. Results of the study carried out by Beauchet and colleagues [1] strongly supported the assumption that gait variability increases as walking speed decreases and, thus, gait might be more unstable when healthy subjects walk slower compared to their preferred walking speed. Additionally, Kang and Dingwell [8] have found potential electromyography markers that related to increased neuromotor noise associated with ageing and may indicate subtle deterioration of gait function that could lead to future functional declines. Apart from these extremely analytical studies that have been focussing on detailed gait analysis as such, we would like, in the framework of our Mobility in Ageing project, to address questions such as: (i) What is the basic structure of gait in different groups of subjects of our interest? (ii) How do these basic gait parameters correlate with the results of other clinical, functional and biomechanical tests? (iii) What is the effect of different training interventions on gait parameters?

In summary, we can say that the results of this pilot study have enabled us to finalize our testing protocol. This will include measurements taken only at the 'preferred' and at the 'very fast speed' of walking, since these two speeds have the highest repeatability. However, only the average of three repetitions at the same walking speed results in a very good repeatability. Finally, as we have obtained comparable values of precision and reproducibility with the SWE measuring method compared to the other two methods and because of the organizational simplicity in its use with all categories of subjects we propose to use SWE in our future studies of the project.

\section{Acknowledgements}

The authors would like to acknowledge the support of: (1) the EU Interreg-IVa project Mobility in Ageing (MOBIL - N_00033) of the Slovak-Austrian crossborder cooperation programme 2007-2013, and (2) the co-financing of the Austrian Federal Minister for Science and Research BM.W_F.

\section{Corresponding Author}

Nejc Sarabon, PhD, University of Primorska, Science and Research Center, Institute for Kinesiological Research. Garibaldijeva 1, SI 6000 Koper, Slovenia. Email: Nejc.sarabon@zrs.upr.si

\section{References}

[1] Beauchet O, Annweiler C, Lecordroch Y, et al. Walking speed-related changes in stride time variability: effects of decreased speed. J Neuroeng Rehabil 2009;6:32.

[2] Chambers HG, Sutherland DH. A practical guide to gait analysis. J Am Acad Orthop Surg 2002;10(3):222-231.

[3] Chung M, Wang MJ. The change of gait parameters during walking at different percentage of preferred walking speed for healthy adults aged 20-60 years. Gait Posture 2010;31(1):131135.

[4] Dietz V. Do human bipeds use quadrupedal coordination? Trends Neurosci 2002;25(9):462467.

[5] Dijkstra B, Zijlstra W, Scherder E, Kamsma Y. Detection of walking periods and number of steps in older adults and patients with Parkinson's disease: accuracy of a pedometer and an accelerometry-based method. Age Ageing 2008;37(4):436-441.

[6] Gramsbergen A. Postural control in man: the phylogenetic perspective. Neural Plast 2005;12(23):77-88; discussion 263-272.

[7] Hausdorff JM. Gait dynamics, fractals and falls: finding meaning in the stride-to-stride fluctuations of human walking. Hum Mov Sci 2007;26(4):555-589.

[8] Kang HG, Dingwell JB. Dynamics and stability of muscle activations during walking in healthy young and older adults. J Biomech 2009;42(14):2231-2237. 


\section{Gait and balance in young and elderly}

European Journal Translational Myology - Basic Applied Myology 2010; 1 (4): 181-186

[9] Kavanagh JJ, Menz HB. Accelerometry: a technique for quantifying movement patterns during walking. Gait Posture 2008;28(1):1-15.

[10] Kuo AD, Donelan JM. Dynamic principles of gait and their clinical implications. Phys Thr 2010;90(2):157-174.

[11] Latash M. Neurophysiological Basis of Movement - 2nd Edition. 2. ed. Human Kinetics; 2007.

[12] Latash M, Zatsiorsky V. Classics in Movement Science. Champaign: Human Kinetics; 2001.

[13] Latt MD, Menz HB, Fung VS, Lord SR. Walking speed, cadence and step length are selected to optimize the stability of head and pelvis accelerations. Exp Brain Res 2008;184(2):201209.

[14] Le Masurier GC, Bauman AE, Corbin CB, et al. Assessing walking behaviors of selected subpopulations. Med Sci Sports Exerc 2008;40(7 Suppl):S594-602.

[15] Lovejoy CO. The natural history of human gait and posture. Part 3. The knee. Gait Posture 2007;25(3):325-341.

[16] Lythgo N, Wilson C, Galea M. Basic gait and symmetry measures for primary school-aged children and young adults. II: Walking at slow, free and fast speed. Gait Posture. 2010. Available at:http://www.ncbi.nlm.nih.gov/pubmed/2097101 3 [Accessed December 19, 2010].

[17] Mackey AH, Stott NS, Walt SE. Reliability and validity of an activity monitor (IDEEA) in the determination of temporal-spatial gait parameters in individuals with cerebral palsy. Gait Posture 2008;28(4):634-639.

[18] Moe-Nilssen R, Aaslund MK, Hodt-Billington C, Helbostad JL. Gait variability measures may represent different constructs. Gait Posture 2010;32(1):98-101.

[19] Newell KM, Deutsch KM, Sosnoff JJ, MayerKress G. Variability in motor output as noise. In:
Movement system variability.Vol 2006. Champaign: Human Kinetics; 2006:3-24.

[20] Pandy MG, Andriacchi TP. Muscle and joint function in human locomotion. Annu Rev Biomed Eng 2010;12:401-433.

[21] Paróczai R, Kocsis L. Analysis of human walking and running parameters as a function of speed. Technol Health Care 2006;14(4-5):251-260.

[22] Patla AE. Adaptive human locomotion: influence of neural, biological and mechanical factors on control mechanisms. In: Clinical disorders of balance, posture and gait. 2004. 2. ed. London: Arnold.

[23] Perry AK, Blickhan R, Biewener AA, Heglund NC, Taylor CR. Preferred speeds in terrestrial vertebrates: are they equivalent? J Exp Biol 1988;137:207-219.

[24] Ryan CG, Grant PM, Tigbe WW, Granat MH. The validity and reliability of a novel activity monitor as a measure of walking. Br J Sports Med 2006;40(9):779-784.

[25] Saremi K, Marehbian J, Yan X, et al. Reliability and validity of bilateral thigh and foot accelerometry measures of walking in healthy and hemiparetic subjects. Neurorehabil Neural Repair 2006;20(2):297-305.

[26] Schmitt D. Insights into the evolution of human bipedalism from experimental studies of humans and other primates. J Exp Biol 2003;206(9):14371448.

[27] Sorsdahl AB, Moe-Nilssen R, Strand LI. Testretest reliability of spatial and temporal gait parameters in children with cerebral palsy as measured by an electronic walkway. Gait Posture 2008;27(1):43-50.

[28] Zehr EP, Duysens J. Regulation of arm and leg movement during human locomotion. Neuroscientist 2004;10(4):347-361. 